\title{
Targeted genomic profiling reveals recurrent KRAS mutations and gain of chromosome $1 q$ in mesonephric carcinomas of the female genital tract
}

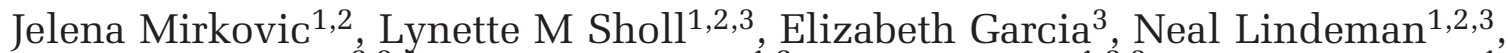 \\ Laura MacConaill ${ }^{2,3,4}$, Michelle Hirsch ${ }^{1,2}$, Paola Dal Cin ${ }^{1,2,3}$, Melissa Gorman ${ }^{1}$, \\ Justine A Barletta ${ }^{1,2}$, Marisa R Nucci ${ }^{1,2}$, McCluggage W Glenn ${ }^{5}$ and Brooke E Howitt ${ }^{1,2}$ \\ ${ }^{1}$ Department of Pathology, Brigham and Women's Hospital, Boston, MA, USA; ${ }^{2}$ Harvard Medical School, \\ Boston, MA, USA; ${ }^{3}$ Center for Advanced Molecular Diagnostics, Brigham and Women's Hospital, Boston, MA, \\ USA; ${ }^{4}$ Dana-Farber Cancer Institute, Boston, MA, USA and ${ }^{5}$ Department of Pathology, Belfast Health and \\ Social Care Trust, Belfast, Northern Ireland, UK
}

\begin{abstract}
Mesonephric carcinoma is a rare form of gynecologic cancer derived from mesonephric remnants usually located in the lateral wall of the uterine cervix. An analogous tumor occurs in the adnexa, female adnexal tumor of probable Wolffian origin. The pathogenesis and molecular events in mesonephric carcinoma are not known. The aim of this study was to examine the molecular alterations in mesonephric carcinoma to identify driver mutations and therapeutically targetable mutations. This study consisted of 19 tumors from 17 patients: 18 mesonephric carcinomas (15 primary tumors and three metastatic tumors) and 1 female adnexal tumor of probable Wolffian origin. In two patients, both primary and metastatic tumors were available. Genomic DNA was isolated and targeted next-generation sequencing was performed to detect mutations, copy number variations, and structural variants by surveying full exonic regions of 300 cancer genes and 113 selected intronic regions across 35 genes. Fluorescence in situ hybridization (FISH) for $1 \mathrm{p}$ and $1 \mathrm{q}$ was performed in two cases. Eighty-one percent $(13 / 16)$ of mesonephric carcinomas had either a $\operatorname{KRAS}(n=12)$ or $\operatorname{NRAS}(n=1)$ mutation. Mutations in chromatin remodeling genes (ARID1A, ARID1B, or SMARCA4) were present in $62 \%$ of mesonephric carcinomas. All mesonephric carcinomas lacked mutations in PIK3CA and PTEN. The most common copy number alteration was 1q gain, found in 12 (75\%) mesonephric carcinomas; this was confirmed by FISH in two cases. Mesonephric carcinoma is characterized by molecular alterations that differ from those of more common variants of cervical and endometrial adenocarcinoma, which harbor KRAS/NRAS mutations in $7 \%$ and $25 \%$ of cases, respectively. KRAS/NRAS mutations are common in mesonephric carcinoma and are often accompanied by gain of $1 \mathrm{q}$ and mutations in chromatin remodeling genes. Targeting inhibitors of the RAS/MAPK pathway may be useful in the treatment of mesonephric carcinoma.

Modern Pathology (2015) 28, 1504-1514; doi:10.1038/modpathol.2015.103; published online 4 September 2015
\end{abstract}

Mesonephric carcinoma is a rare malignant tumor of the female genital tract that occurs most commonly in the uterine cervix, representing $<1 \%$ of all cervical carcinomas. ${ }^{1-3}$ Primary mesonephric carcinomas of the uterine corpus and the vagina

Correspondence: Dr BE Howitt, MD, Department of Pathology, Brigham and Women's Hospital, 75 Francis Street, Boston, MA 02115, USA.

E-mail: bhowitt@partners.org

Received 15 June 2015; revised 29 July 2015; accepted 30 July 2015; published online 4 September 2015 have also been described. ${ }^{4-8}$ Patients with mesonephric carcinoma usually present with abnormal vaginal bleeding (in $\sim 60-70 \%$ of the cases), abnormal Papanicolaou smear, a cervical mass on pelvic examination, or the tumor can be discovered incidentally. ${ }^{1,2}$ Grossly, these tumors are frequently in the form of an exophytic, nodular, or polypoid friable mass usually in the lateral wall of the cervix or involving the cervical wall circumferentially. They can also diffusely and circumferentially enlarge the cervical wall without a discernible mass, or can even be grossly unremarkable. ${ }^{1,2}$ 
Histologically, mesonephric carcinomas are characterized by a variety of architectural patterns such as tubular, ductal, retiform, solid, spindle cell, and sex-cord like. An admixture of patterns is frequently present in the same tumor. Because of the wide range of appearances, the differential diagnosis of mesonephric carcinoma can be broad, and include benign mesonephric hyperplasia, endometrioid adenocarcinoma, clear cell carcinoma, carcinosarcoma, and serous carcinoma among others. As mesonephric hyperplasia is often found at the periphery of the tumor or admixed with it, ${ }^{1,2,9,10}$ mesonephric carcinomas are thought to be derived from mesonephric remnants and/or hyperplasia. Similarly, mesonephric remnants in the adnexa are thought to give rise to the female adnexal tumor of probable Wolffian origin. These neoplasms, which are exceedingly rare, occur mainly in the para-ovarian region but also occasionally within the ovary and are presumed to be of mesonephric (Wolffian) origin based on the location, morphologic dissimilarity to other ovarian tumors, and some ultrastructural and immunohistochemical homology to the mesonephric duct. ${ }^{11}$

The pathogenesis and molecular features of mesonephric carcinoma and female adnexal tumor of probable Wolffian origin have not been reported in the literature. The purpose of this study was to characterize the mutations and copy number alterations characteristic of mesonephric carcinoma using a targeted next-generation sequencing (NGS) assay to identify driver mutations, therapeutically targetable mutations, and diagnostically useful molecular alterations. We report the findings in a series of mesonephric carcinoma of the female genital tract and one female adnexal tumor of probable Wolffian origin.

\section{Materials and methods}

\section{Case Selection}

Following approval by the Institutional Review Board at Brigham and Women's Hospital in Boston, MA, USA, the BWH pathology files were searched for cases of mesonephric carcinoma. All Brigham and Women's Hospital cases of mesonephric carcinoma with available formalin-fixed paraffinembedded material and histologically confirmed diagnoses were included in the study, and six additional mesonephric carcinomas and one female adnexal tumor of probable Wolffian origin were contributed by one of the authors (WGM). In all, 19 tumors from 17 subjects were included in the study: 18 mesonephric carcinomas from 16 patients (13 cervical primary tumors, 2 uterine corpus primary tumors, and 3 metastatic tumors), and 1 female adnexal tumor of probable Wolffian origin. For 16 cases, only one to two representative H\&E slides were available for histological review; in the remainder of cases, all slides were available for review.
Thirteen of these cases have been previously reported by Kenny et $a l^{7}$ and Howitt et $a l^{12}$ (see Table 1).

\section{Targeted Hybridization Capture NGS}

Samples were processed in the Department of Pathology, in the Center for Advanced Molecular Diagnostics at Brigham and Women's Hospital, a CLIA-certified clinical laboratory, for all stages of the NGS assay. Unstained 4-micron formalin-fixed paraffin-embedded tissue sections of tumor were manually scraped for all cases and DNA was isolated using a commercially available kit (Qiagen, Valencia, CA), following the manufacturer's instructions. Paired germline samples were not analyzed. DNA was quantified (PicoGreen), and samples with at least $50 \mathrm{ng} / \mathrm{ul}$ of DNA proceeded to library preparation. Hybrid capture libraries were prepared following previously published protocols. ${ }^{13,14}$ Sheared DNA was hybridized to a set of custom-designed capture probes (Agilent SureSelect) targeting the complete exonic regions of 300 oncogenes and tumor-suppressor genes and 113 intronic regions across 35 genes for the evaluation of structural rearrangements (complete list of genes available in Supplementary File 1). Sequencing was performed using Illumina HiSeq 2500. Data were analyzed by an internally developed bioinformatics pipeline composed of reconfigured publically available tools and internally developed algorithms (VisCap Cancer, Phaser, BreaKmer). ${ }^{13}$ Pooled sample reads were demultiplexed using Picard (http://picard.source forge.net/command-line-overview.shtml), aligned to Human Genome Reference Consortium reference sequence GRCh $37 \mathrm{p} 13^{15}$ and duplicate reads were removed. GATK ${ }^{16}$ was used to refine alignments around insertion/deletion (indel) sites. Single nucleotide variants were called using MuTect ${ }^{17}$ and indels using Indelocator (http://www.broadinstitute. org/cancer/cga/indelocator). Annotation was performed using Oncotator. ${ }^{18}$ Because tumor tissues were tested without a paired normal from individual patients, additional informatics steps were taken to identify common single nucleotide polymorphisms: any single nucleotide polymorphism present at $>0.1 \%$ in Exome Variant Server, NHLBI GO Exome Sequencing Project, Seattle, WA (URL: http://evs.gs. washington.edu/EVS/, accessed 4/1/2015) was filtered, however, variants also present in the Catalogue of Somatic Mutations in Cancer (COSMIC; cancer.sanger.ac.uk) were rescued for manual review. VisCap Cancer calls copy number changes based on $\log 2$ ratios that are calculated using a normalized depth of coverage against a median from a panel of normal (non-cancer) samples. Circular binary segmentation was used to segment the data; segments were called via strict thresholding. Unique, aligned (hg19) sequence reads with PHRED $>30$ were reviewed, annotated, and interpreted using 
Table 1 Clinicopathologic features of mesonephric tumors

\begin{tabular}{|c|c|c|c|c|c|c|c|c|c|}
\hline Case \# & Patient age & Tumor site & $\begin{array}{l}\text { Tumor } \\
\text { size }\end{array}$ & $\begin{array}{l}\text { Associated with } \\
\text { mesonephric hyperplasia? }\end{array}$ & $\%$ Solid & $\begin{array}{l}\text { Stage at } \\
\text { diagnosis }\end{array}$ & $\begin{array}{l}\text { Time to last } \\
\text { follow-up }\end{array}$ & Status at last follow-up & $\begin{array}{l}\text { Previously published } \\
\text { (reference number) }\end{array}$ \\
\hline 1 & 76 & Cervix & $2.2 \mathrm{~cm}$ & Yes & $5 \%$ & FIGO 2B & - & - & 7,12 \\
\hline 2 & 56 & Cervix & - & Yes & $0 \%$ & FIGO 1B1 & 67 months & $\begin{array}{l}\text { Alive, no evidence of } \\
\text { disease }\end{array}$ & - \\
\hline 3 & 67 & Cervix & - & Yes & $0 \%$ & FIGO 1B2 & - & - & 7,12 \\
\hline 4 & 47 & Cervix & - & No & $80 \%$ & FIGO 2B & - & - & 7,12 \\
\hline $5 \mathrm{~A}$ & 38 & Cervix & - & - & $15 \%$ & FIGO 3B & 10 months & $\begin{array}{l}\text { Alive with disease } \\
\text { (abdominal, pelvic, and } \\
\text { liver) }\end{array}$ & - \\
\hline $5 B$ & & Ovary (metastasis) & & & & & & & \\
\hline 6 & 47 & Uterine corpus & - & - & $50 \%$ & FIGO $3 \mathrm{~A}$ & - & - & 7,12 \\
\hline 7 & 51 & Cervix & - & Yes & $0 \%$ & FIGO 3B & 149 months & $\begin{array}{l}\text { Alive with disease (lung } \\
\text { nodules at } 120 \text { months) }\end{array}$ & 12 \\
\hline 8 & 64 & Cervix & $4.5 \mathrm{~cm}$ & - & $60 \%$ & FIGO 1B2 & 36 months & $\begin{array}{l}\text { Alive, no evidence of } \\
\text { disease }\end{array}$ & 12 \\
\hline 9 & 67 & Cervix & $1 \mathrm{~cm}$ & Yes & $40 \%$ & Unknown & - & - & 12 \\
\hline 10 & 71 & Cervix & - & Yes & $0 \%$ & FIGO 2B & - & - & 7,12 \\
\hline 11 & 81 & $\begin{array}{l}\text { Unknown (ovary } \\
\text { metastasis) }\end{array}$ & $11 \mathrm{~cm}$ & - & $5 \%$ & Unknown & - & - & - \\
\hline $12 \mathrm{~A}$ & 65 & Uterine corpus & $9 \mathrm{~cm}$ & - & $30 \%$ & FIGO 3 A & 19 months & $\begin{array}{l}\text { Alive with disease (lung } \\
\text { metastases at } 1 \text { month) }\end{array}$ & 12 \\
\hline $12 \mathrm{~B}$ & & Lung (metastasis) & & & $0 \%$ & & & & \\
\hline 13 & 54 & Cervix & $12 \mathrm{~cm}$ & Yes & $90 \%$ & Unknown & - & - & 12 \\
\hline 14 & 74 & Cervix & $3.1 \mathrm{~cm}$ & Yes & $0 \%$ & FIGO 1B1 & - & - & 12 \\
\hline 15 & 48 & Cervix & - & Yes & $5 \%$ & Unknown & - & - & 12 \\
\hline 16 & 37 & Cervix & - & - & $30 \%$ & Unknown & - & - & - \\
\hline 17 & 42 & Adnexa & $20 \mathrm{~cm}$ & - & - & FIGO 1 A & - & - & 7 \\
\hline
\end{tabular}

Cases 1-16 are mesonephric carcinoma, and case 17 is a female adnexal tumor of probable Wolffian origin. " - " indicates that no data were available. 
Integrated Genome Viewer (Broad Institute, Cambridge, MA) and a suite of internally developed Web-based tools. Samples with a mean target coverage of $<50 \times$ were failed and excluded from further analysis. Individual variants present at $<10 \%$ allele fraction or in regions with $<50 \times$ coverage were flagged for manual review and interpreted by the reviewing laboratory scientists and molecular pathologists based on overall tumor percentage, read depth, complexity of alteration, and evidence for associated copy number alterations.

Gene mutation frequency calculations were analyzed and reported per subject, with a secondary analysis of matched primary and metastatic tumors. The frequency of the most commonly mutated genes in mesonephric carcinoma identified in our study was compared with conventional cervical adenocarcinomas $^{19}$ and endometrial adenocarcinomas $^{20}$ and as reported in The Cancer Genome Atlas (TCGA) Research Network (http://cancergen ome.nih.gov/).

\section{Fluorescence In Situ Hybridization}

FISH was performed on $4-\mu$ m-thick formalin-fixed paraffin-embedded sections from cases with sufficient material, according to standard protocols. Specifically, gain of the long arm of chromosome 1 (1q) was evaluated using the Vysis LSI1p36 and LSI1q25 Dual Color Probe Set 1 (Abbott Molecular, Des Plaines, IL) at $1 \mathrm{p} 36$ and 1q25, respectively, on both tumor and non-neoplastic tissue (the non-neoplastic tissue served as internal positive control). Probes and nuclei were co-denatured simultaneously, followed by hybridization and washing, according to the manufacturer's directions. The ratio of hybridization signals for 1p36 and 1q25 was calculated on 50 nuclei for each case.

\section{Results}

\section{Clinicopathologic Features of the Cohort}

Our study comprised 19 tumors from 17 patients (16 mesonephric carcinomas and 1 female adnexal tumor of probable Wolffian origin); the clinicopathologic features are summarized in Table 1. Two patients had matched primary and metastatic tumor samples: one uterine corpus primary with a lung metastasis (case 12A/B) and one cervical primary with an ovarian metastasis (case 5A/B). One case had only metastatic mesonephric carcinoma involving the ovary available for testing (case 11). Histologically, the mesonephric carcinomas were characterized by a variety of architectural patterns including tubular, ductal, retiform, cribriform, and spindled/ solid (Figure 1). Two or more patterns were frequently observed in the same tumor. The majority of tumors contained some degree of spindled/solid areas $(13 / 19 ; 68 \%)$, ranging from 5 to $90 \%$ of the tumor in the sections available for review. All cases from patients with confirmed metastasis contained at least focal areas of spindled/solid morphology (ranging from 5 to $30 \%$ ). Associated mesonephric hyperplasia was confirmed in 9/16 (56\%) mesonephric carcinoma.

\section{Molecular genetic findings}

\section{Quality Assurance Parametrics in the NGS Assay}

Across all 19 tumors, the mean number of unique, high quality aligned reads was 5168690 (range 4 109 829-7 579 048), with a mean target coverage of 132 (range 95-194). Overall, 94-99\% of targeted sequences had at least $30 \times$ target base coverage across the cases.

\section{Overview of Single Nucleotide Variations and Copy Number Variations Identified in Mesonephric Carcinoma}

A complete list of the single nucleotide variants identified across all tumors is available in Supplementary File 2. Overall, 117 single nucleotide variations in 75 genes were identified across all mesonephric carcinomas $(n=16)$ and comprised 93 missense, 10 nonsense, 7 frameshift, 6 indels, and 1 splice site mutation. Of the missense mutations, 37 had been previously reported in other tumor types and confirmed as somatic mutations in COSMIC (cancer.sanger.ac.uk), and 56 had not been previously reported as somatic mutations and thus were classified as novel. The mean number of single nucleotide variations per tumor was 7.1 (range 2-13). The recurrent single nucleotide variations are shown in Figure 2 and are discussed below in further detail. Copy number changes in mesonephric carcinoma were primarily whole chromosome or arm level alterations, with very few gene level copy number variations. No high level amplifications or two copy (homozygous) deletions were identified. Five cases had no copy number variations. Copy number variations at the chromosomal arm level are summarized in Figure 3a.

\section{KRAS and NRAS Activating Mutations Are Common in Mesonephric Carcinoma}

Twelve of 16 mesonephric carcinomas harbored activating KRAS mutations (all missense; $6 \mathrm{G} 12 \mathrm{~V}, 4$ G12D, $1 \mathrm{G} 12 \mathrm{C}$, and $1 \mathrm{Q} 61 \mathrm{H})$, including case $5 \mathrm{~A} / \mathrm{B}$ with a cervical primary and ovarian metastasis, and case $12 \mathrm{~A} / \mathrm{B}$ with a uterine corpus primary and lung metastasis. One mesonephric carcinoma had an activating NRAS mutation (missense, Q61R). KRAS and NRAS mutations were mutually exclusive. In total, 13/16 (81\%) mesonephric carcinomas had either a $\operatorname{KRAS}(n=12)$ or NRAS $(n=1)$ mutation. One mesonephric carcinoma (case 16) that lacked 

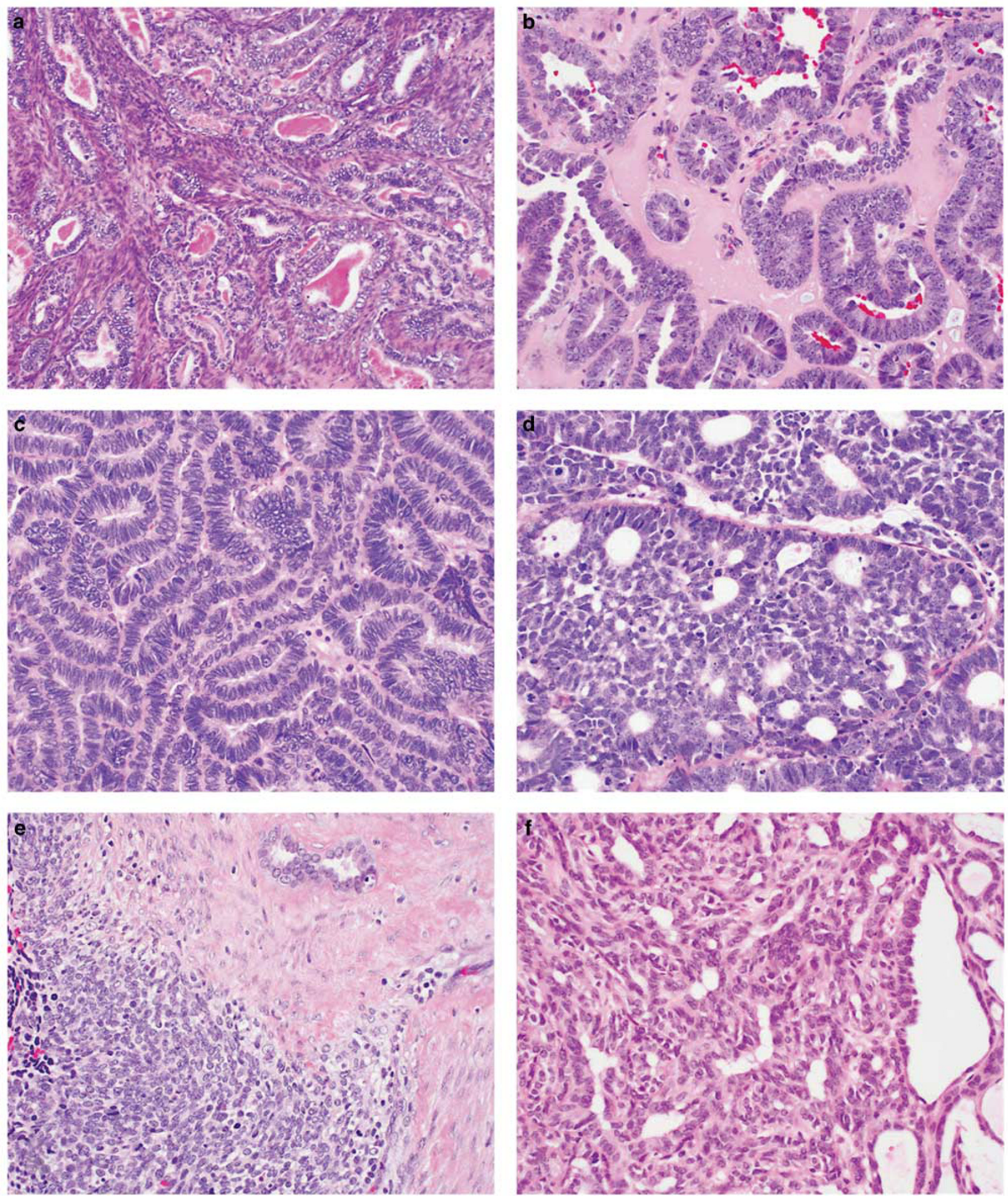

Figure 1 Histologic features of mesonephric carcinoma and female adnexal tumor of probable Wolffian origin. Brightly eosinophilic secretory material (a) may be seen in tubule lumens. Tubular architecture and hyalinized stroma (b) is common in mesonephric carcinoma. Frequent morphologic patterns include ductal (c) and cribriform (d). Solid or spindled foci were present in a subset of cases, and as shown in this example, often also associated with tubule formation (e). The single female adnexal tumor of probable Wolffian origin in this series demonstrates morphologic similarity to mesonephric carcinoma (f). 


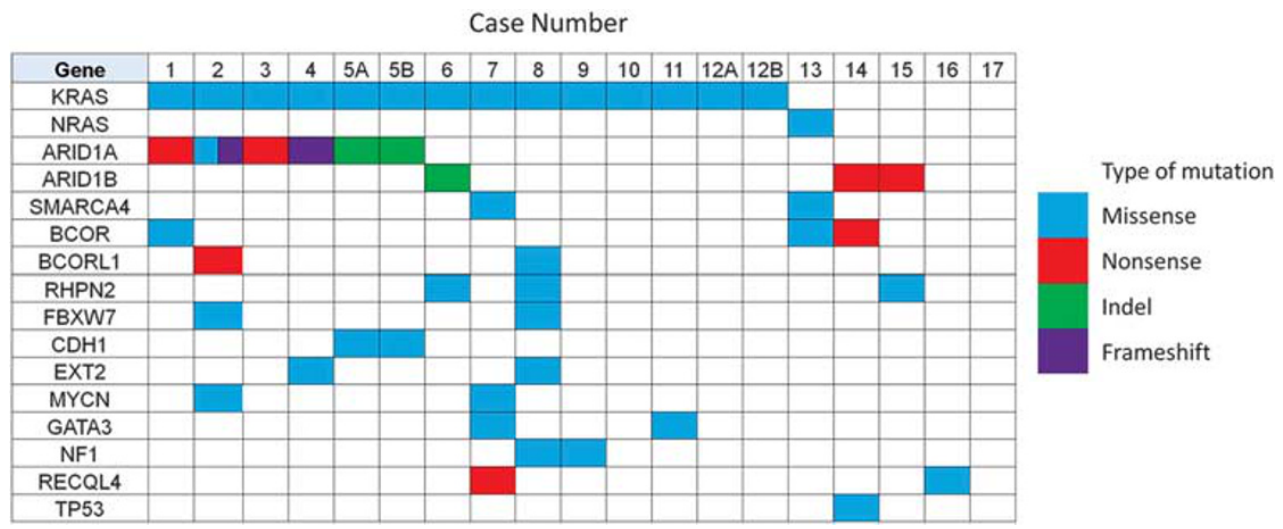

Figure 2 Recurrent single nucleotide variations in mesonephric tumors. All genes with mutations in at least two cases are included. Each column is a case number and each row represents a gene. Blue indicates a missense mutation, red indicates a nonsense mutation, green indicates an indel, and purple indicates a frameshift mutation. A white block indicates no single nucleotide variation was identified in that case/gene.

KRAS or NRAS mutation had evidence of gene level copy number gains of HRAS. No copy number gains of KRAS or NRAS were present in any case.

\section{Alterations in the Chromatin Remodeling Genes ARID1A, ARID1B, and SMARCA4}

Five mesonephric carcinomas harbored six ARID1A mutations (one indel, two nonsense, two frameshift, and one missense) including case $12 \mathrm{~A} / \mathrm{B}$ with the uterine corpus primary and ovarian metastasis. Of the five mesonephric carcinomas with ARID1A mutations, one (case 2) had two different ARID1A mutations, and three (cases 1, 3, and 5) also had evidence of copy number loss at ARID1A, raising the possibility of bi-allelic inactivation. One uterine corpus and two cervical mesonephric carcinomas harbored ARID1B mutations (two nonsense and one indel), one of which also showed copy number loss at $A R I D 1 B$ (case 6). Two mesonephric carcinomas harbored mutations in SMARCA4 (one splice site (case 7) and one missense (case 13)), neither of which had evidence of copy number loss. In total, 10/16 (62\%) mesonephric carcinomas harbored ARID1A, ARID1B, or SMARCA4 mutations, which were all mutually exclusive.

\section{Mutations in BCOR/BCORL1 Genes}

Three mesonephric carcinomas harbored BCOR mutations (two missense and one nonsense; cases 1, 13, and 14), and two mesonephric carcinomas harbored three BCORL1 mutations (two nonsense (case 2) and one missense (case 8)). In total, $5 / 16 \quad(31 \%)$ mesonephric carcinomas contained BCOR or BCORL1 mutations.

\section{TP53 Mutations Are Uncommon in Mesonephric Carcinoma}

Only one tumor in this series (cervical mesonephric carcinoma; case 14) harbored a mutation in TP53 gene (missense, M237I) and was not accompanied by copy number loss of TP53 or $17 \mathrm{p}$. This case also harbored an ARID1B mutation but lacked KRAS or NRAS mutations.

\section{Gain of 1q}

Twelve of $16(75 \%)$ mesonephric carcinomas had gain of $1 \mathrm{q}$ by copy number analysis (Figure $3 \mathrm{~b}$ ), which was confirmed by FISH in 2 cases with the 1q25 signals approximately twice the number of signals observed for $1 \mathrm{p} 36$ signals (three to seven copies of $1 \mathrm{q}$ in case 12 , and two to five copies of $1 \mathrm{q}$ in case 5) (Figure 3c). Material was unavailable for FISH studies in the remainder of cases. Seven of the cases with 1q gain also had evidence of loss of $1 p$ by copy number variation analysis.

\section{Other Recurrent Copy Number Variations in Mesonephric Carcinoma}

Mesonephric carcinomas were characterized by other recurrent copy number variations including $2 p$ gain (three cases), $9 p$ or chromosome 9 loss (five cases), chromosome 19 gain (three cases), chromosome 19 loss (two cases), and chromosome 10, 12, and 20 gain (five, five, and six cases, respectively).

\section{Translocations in Mesonephric Carcinoma}

Case 6 harbored a novel translocation: MDM4-SNX9 (MDM4 on chromosome 1q32.1, exons 1-6; position 204507547; SNX9 on chromosome 6q25.3, exons 3-18; position 158289657). As this alteration was 
a

Chromosome arm

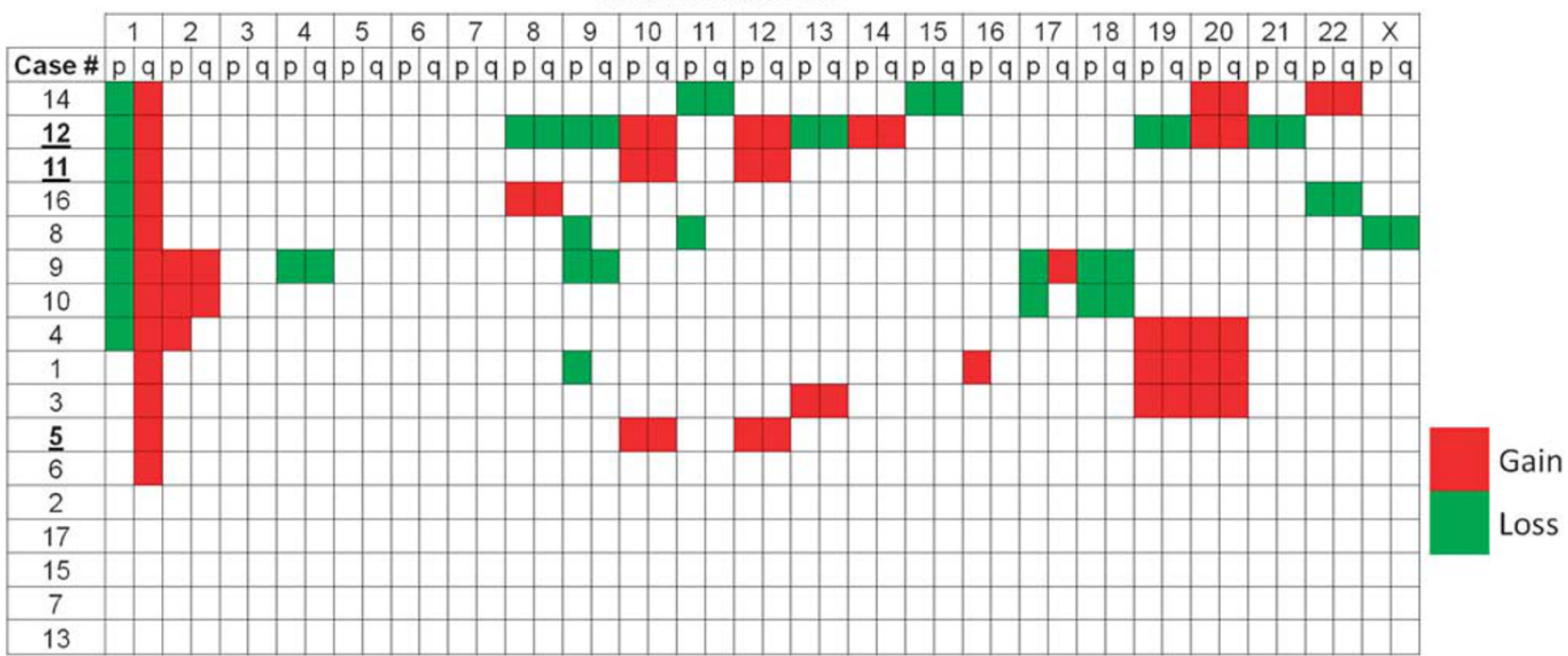

b
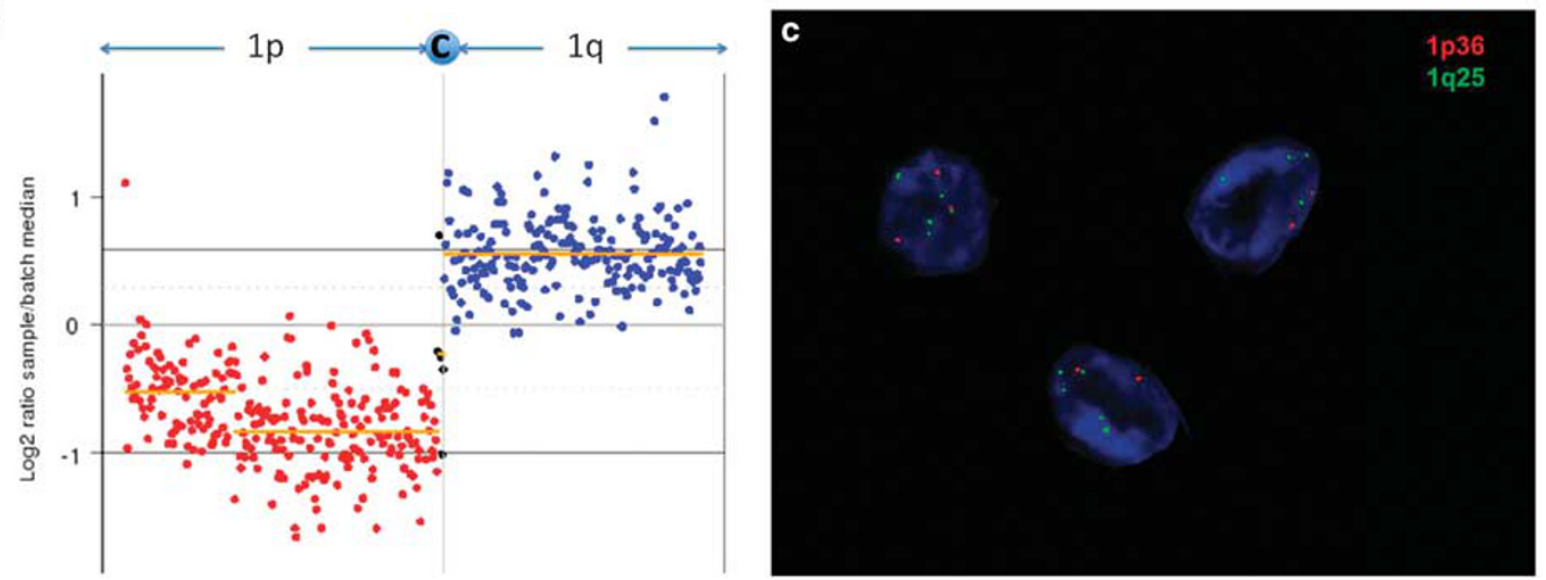

Figure 3 (a) Recurrent copy number alterations in mesonephric tumors. Blocks highlighted in red indicate copy number gains, those highlighted in green indicate copy number loss. Blank (white) blocks indicate copy number neutral genes. Case numbers are listed in the left hand column, with cases associated with tumor metastasis in bold and underlined text. (b) Copy number variation plot of chromosome 1, with evidence for gain of the entire long (q) arm, and in a subset of cases, loss of the majority of the short (p) arm. (c) Fluorescence in situ hybridization for $1 \mathrm{p}$ and $1 \mathrm{q}$ show gain of $1 \mathrm{q}$ (green) relative to numbers of $1 \mathrm{p}$ (red), with two to three copies of $1 \mathrm{p} 36$ and five to seven copies of $1 \mathrm{q} 25$.

identified in only one tumor without additional material available, no further confirmatory testing was performed.

\section{Correlation of Morphologic/Clinical Features with Molecular Alterations Including Matched Primary and Metastatic Tumors}

All cases from patients with confirmed metastasis (cases $5 \mathrm{~A} / \mathrm{B}, 7,11$, and $12 \mathrm{~A} / \mathrm{B}$ ) had a KRAS mutation. Both cases with matched primary and metastatic tumors had KRAS G12D mutation in both the primary and the metastatic tumor. The case with ovarian metastasis (no material was available from the primary tumor) had a KRAS G12V mutation. Case $5 \mathrm{~A} / \mathrm{B}$ harbored the same ARID1A mutation in both the cervical primary and the ovarian metastasis. BCOR, BCORL1, and TP53 mutations were not present in any of the tumors from patients with confirmed metastasis. Although most of the metastatic tumors exhibited gains of chromosomes 10 and 12 (cases $5 \mathrm{~A} / \mathrm{B}, 11$, and $12 \mathrm{~A} / \mathrm{B}$ ), none of the cases without metastases had this finding. Of note, one patient (case \#7; lacking gain of chromosome 10 and 12) developed lung nodules 120 months after the diagnosis of mesonephric carcinoma; and by report, the biopsy revealed metastatic carcinoma. In considering morphologic features of mesonephric carcinoma, specifically the presence and percentage of solid/spindled component, we found no correlation between molecular events in cases with and without a spindled/solid component. 


\section{Molecular Features of Female Adnexal Tumor of Probable Wolffian Origin in Comparison with Mesonephric Carcinoma}

The single female adnexal tumor of probable Wolffian origin (case 17) was characterized by three single nucleotide variations including PRKDC H1069Y, FANCA H227D, and GNAS frameshift (I2fs) mutations. The female adnexal tumor of probable Wolffian origin did not demonstrate any copy number variations. There were no overlapping molecular alterations between the female adnexal tumor of probable Wolffian origin and mesonephric carcinoma.

\section{Discussion}

Mesonephric carcinoma is a malignant gynecologic tumor derived from mesonephric remnants and/or hyperplasia. In males, the mesonephric ducts give rise to the efferent ducts of the testis, the epididymis, the vasa deferentia, the seminal vesicles, and the ejaculatory ducts. In females, the mesonephric ducts undergo regression; however, mesonephric remnants may persist in the broad ligaments, lateral wall of the uterus (most notably in the cervix), and vagina. ${ }^{9}$ These remnants are presumed to be the origin of mesonephric carcinoma. Similarly, mesonephric remnants in the adnexa are presumed to be the origin of female adnexal tumor of probable Wolffian origin. ${ }^{11,21}$

Because of the wide range of morphological appearances of mesonephric carcinoma, often with an admixture of tubular, ductal, retiform, solid, spindle cell, and sex-cord like elements, the differential diagnosis can be broad, and includes florid mesonephric hyperplasia, endometrioid adenocarcinoma, endocervical adenocarcinoma, clear cell carcinoma, carcinosarcoma, and serous carcinoma, among other neoplasms. The admixture of different morphological patterns may be a clue to the diagnosis of mesonephric carcinoma. Although immunohistochemistry can be helpful in the differential diagnosis, ${ }^{1,7,12,22-25}$ there is no definitive marker for mesonephric carcinoma. GATA3 has recently been shown to be quite a sensitive and specific marker of mesonephric epithelia, particularly mesonephric remnants and hyperplasia; however, the staining pattern is less reliable in mesonephric carcinoma, especially when solid or spindled patterns are present. ${ }^{12,25}$ Other markers that may be positive in mesonephric carcinoma include CD10 and calretinin while estrogen and progesterone receptor are usually negative. ${ }^{1,22,26,27}$ However, none of these markers is sufficiently sensitive or specific for mesonephric carcinoma to be reliable in diagnosis. Therefore, identifying characteristic molecular alterations may be useful in establishing a diagnosis of mesonephric carcinoma, considering that the most common differential diagnoses, endocervical and endometrial adenocarcinomas of other morphological types, have been well characterized at the molecular level.

In this study, we evaluated 18 mesonephric carcinomas from 16 patients and 1 female adnexal tumor of probable Wolffian origin using a targeted NGS assay including a large panel of wellestablished oncogenes and tumor-suppressor genes to identify molecular alterations in mesonephric carcinoma. Our study found that mesonephric carcinoma is characterized by a limited number of molecular aberrations that may be diagnostically useful and therapeutically important. The common mutations in mesonephric carcinoma are significantly different from those reported in other cervical and endometrial adenocarcinomas ${ }^{19,20,28,29}$ and in the TCGA datasets by accessing the cBioPortal for Cancer Genomics, ${ }^{30,31}$ (www.cbioportal.org). For example, KRAS/NRAS mutations are the most common molecular aberration detected in mesonephric carcinoma (81\%); in contrast, these mutations are less common in other cervical and endometrial adenocarcinomas (less than 30\%), ${ }^{32-34}$ particularly in the microsatellite stable endometrioid adenocarcinomas and the serous endometrial carcinomas. The chromatin remodeling genes ARID1A, ARID1B, and SMARCA4 are frequently mutated in mesonephric carcinoma (31, 19, 12\%, respectively; overall $62 \%$ ). In contrast, these genes are uncommonly mutated in other cervical carcinomas. In endometrial adenocarcinomas, the frequency of ARID1A mutations is comparable with that in mesonephric carcinoma, but $A R I D 1 B$ and SMARCA4 are less commonly mutated $(4 \%$ and $6 \%$, respectively). Mesonephric carcinomas also commonly harbor BCOR/BCORL1 mutations (19\% and $12 \%$, respectively; combined 31\%). Similarly, BCOR and BCORL1 genes are mutated in $12 \%$ and $8 \%$ of endometrial adenocarcinomas, respectively, but these mutations are uncommon in cervical carcinomas (3\% and $3 \%$, respectively). On the other hand, some of the more commonly mutated genes in endometrial and/or cervical adenocarcinomas include PIK3CA (24-57\% and 16-25\%, respectively) and PTEN ( $>60 \%$ for endometrioid endometrial adenocarcinomas and $8 \%$ for cervical adenocarcinomas), and although these genes were included in our NGS assay, only wild-type sequences of PIK3CA and PTEN were identified in all cases of mesonephric carcinoma. TP53 is uncommonly mutated in mesonephric carcinoma (6\%) and other cervical adenocarcinomas (4\%), while $28 \%$ of endometrial adenocarcinomas (and $>90 \%$ of endometrial serous carcinomas) harbor mutations in TP53. Thus, the lack of PIK3CA, PTEN, and TP53 mutations, in combination with KRAS or NRAS mutation, would support the diagnosis of mesonephric carcinoma over another morphologic type of endometrial or endocervical adenocarcinoma.

Copy number changes in mesonephric carcinoma were primarily at the chromosome or arm level, with 
no distinct gene level copy number changes. No high level amplifications or two copy deletions were identified. The most common copy number variation was gain of 1q, which was present in $75 \%$ of mesonephric carcinoma, and was accompanied by loss of $1 p$ in seven cases. Because gain of $1 \mathrm{q}$ was a recurrent finding in this series, FISH was undertaken to confirm the finding in two cases with available material. Interestingly, in the TCGA data, ${ }^{20} 1 \mathrm{q}$ amplification was very common $(100 \%)$ in a subset of endometrioid adenocarcinomas (somatic copy number alteration Cluster 3), which also had frequent gains of chromosomes 10 and 12 and displayed poor clinical outcome compared with other endometrioid tumor clusters. Similarly, in mesonephric carcinoma, gain of chromosome $1 \mathrm{q}$ as well as 10 and/or 12 may be an indicator of aggressive behavior, as all tumors with this alteration were from patients with confirmed metastasis.

Our findings may have clinical implications for the treatment of patients with mesonephric carcinoma. First, the lack of PI3K pathway alterations in mesonephric carcinomas suggests that PI3K inhibitors may not be effective in the treatment of these neoplasms. Mutations of KRAS result in constitutive activation of mitogen-activated protein kinase (MAPK), also known as extracellular signal-regulated protein kinase, which subsequently activates a variety of downstream cellular and nuclear targets that lead to expression of genes involved in proliferation, differentiation, and survival. $^{35,36}$ Targeted inhibitors of the RAS/MAPK pathway have been evaluated in the treatment of KRAS-mutated carcinomas. ${ }^{37,38}$ The consequences of RAS/MAPK pathway inhibition in ovarian cancer cells have been investigated using a selective inhibitor of MAPK kinase (MEK), CI-1040. Profound growth inhibition and apoptosis were observed in CI-1040-treated ovarian cancer cells with mutations in either KRAS or BRAF, compared with ovarian cancer cells containing wild-type $K R A S / B R A F .^{39}$ Although KRAS-mutated lung cancers, which comprise $20-30 \%$ of non-small cell lung cancers, are known not to respond to targeted therapies, recently it was shown that the combination of PI3K and MEK inhibitors may be a very potent combination in the treatment of KRAS-mutated lung cancers. When mouse models of lung cancer driven by mutant KRAS were treated with a combination of pan-PI3K, a mammalian target of rapamycin inhibitor, and an MEK inhibitor, there was marked synergy in shrinking these tumors. ${ }^{40-42}$ Sensitivity to MEK inhibition has been investigated in pancreatic cancer cell lines with specific KRAS mutations showing promising therapeutic results. ${ }^{43}$ Because $81 \%$ of mesonephric carcinomas harbor KRAS/NRAS mutations, targeted inhibitors of the RAS/MAPK pathway could potentially be useful in the treatment of mesonephric carcinoma. Future studies to determine the efficacy of RAS/MAPK inhibition in mesonephric carcinoma are warranted.
The literature suggests that mesonephric carcinomas without a spindled/solid component present at an earlier stage and have a better prognosis than tumors with a spindled/solid component (these have also been referred to as mesonephric carcinosarcoma), which may present in advanced stage and are clinically more aggressive. ${ }^{6}$ Our study found no correlation between the molecular features of mesonephric carcinoma and presence of a spindled/solid component, but this could potentially be attributed to the fact that most of the cases had only one slide of tumor available for review, which may have not been representative of the overall morphological appearances of the neoplasm.

The relationship between mesonephric carcinoma and female adnexal tumor of probable Wolffian origin remains to be elucidated. None of the recurrent alterations found in mesonephric carcinomas in our study were identified in the single case of female adnexal tumor of probable Wolffian origin. However, obviously no firm conclusions can be drawn given that there was only a single female adnexal tumor of probable Wolffian origin available for our study.

In summary, we have shown that mesonephric carcinoma is characterized by molecular aberrations which are significantly different from those found in other subtypes of cervical and endometrial carcinomas. KRAS/NRAS mutations are common $(81 \%)$ in mesonephric carcinoma. Mesonephric carcinomas are also characterized by gain of $1 \mathrm{q}$ and frequent mutations in chromatin remodeling genes, while TP53 mutations are uncommon and PIK3CA and PTEN mutations were not identified. Gain of chromosome 10 and/or 12 may be an indicator of aggressive behavior in mesonephric carcinoma. Our study suggests that targeted inhibitors of the RAS/ MAPK pathway may be useful in the treatment of mesonephric carcinoma and future studies are warranted to determine their efficacy.

\section{Acknowledgments}

This work was funded by the Department of Pathology, Brigham and Women's Hospital. We thank the staff at the Center for Advanced Molecular Diagnostics for their assistance with this project.

\section{Disclosure/conflict of interest}

The authors declare no conflict of interest.

\section{References}

1 Silver SA, Devouassoux-Shisheboran M, Mezzetti TP et al. Mesonephric adenocarcinomas of the uterine cervix: a study of 11 cases with immunohistochemical findings. Am J Surg Pathol 2001;25:379-387. 
2 Clement $\mathrm{PB}$, Young $\mathrm{RH}$, Keh $\mathrm{P}$ et al. Malignant mesonephric neoplasms of the uterine cervix. A report of eight cases, including four with a malignant spindle cell component. Am J Surg Pathol 1995;19:1158-1171.

3 Buntine DW. Adenocarcinoma of the uterine cervix of probable Wolffian origin. Pathology 1979;11:713-718.

4 Wani Y, Notohara K, Tsukayama C. Mesonephric adenocarcinoma of the uterine corpus: a case report and review of the literature. Int J Gynecol Pathol 2008;27:346-352.

$5 \mathrm{Wu} \mathrm{H}$, Zhang L, Cao W et al. Mesonephric adenocarcinoma of the uterine corpus. Int J Clin Exp Pathol 2014;7:7012-7019.

6 Bague S, Rodriguez IM, Prat J. Malignant mesonephric tumors of the female genital tract: a clinicopathologic study of 9 cases. Am J Surg Pathol 2004;28:601-607.

7 Kenny SL, McBride HA, Jamison J et al. Mesonephric adenocarcinomas of the uterine cervix and corpus: HPV-negative neoplasms that are commonly PAX8, CA125, and HMGA2 positive and that may be immunoreactive with TTF1 and hepatocyte nuclear factor 1beta. Am J Surg Pathol 2012;36:799-807.

8 Roma AA. Mesonephric carcinosarcoma involving uterine cervix and vagina: report of 2 cases with immunohistochemical positivity For PAX2, PAX8, and GATA-3. Int J Gynecol Pathol 2014;33:624-629.

9 Ferry JA, Scully RE. Mesonephric remnants, hyperplasia, and neoplasia in the uterine cervix. A study of 49 cases. Am J Surg Pathol 1990;14:1100-1111.

10 Valente PT, Susin M. Cervical adenocarcinoma arising in florid mesonephric hyperplasia: report of a case with immunocytochemical studies. Gynecol Oncol 1987;27: 58-68.

11 Tiltman AJ, Allard U. Female adnexal tumours of probable Wolffian origin: an immunohistochemical study comparing tumours, mesonephric remnants and paramesonephric derivatives. Histopathology 2001;38: 237-242.

12 Howitt BE, Emori MM, Drapkin R et al. GATA3 is a sensitive and specific marker of benign and malignant mesonephric lesions in the lower female genital tract. Am J Surg Pathol; e-pub ahead of print 11 May 2015.

13 Abo RP, Ducar M, Garcia EP et al. BreaKmer: detection of structural variation in targeted massively parallel sequencing data using kmers. Nucleic Acids Res 2015;43:e19.

14 Wagle N, Berger MF, Davis MJ et al. High-throughput detection of actionable genomic alterations in clinical tumor samples by targeted, massively parallel sequencing. Cancer Discov 2012;2:82-93.

15 Li H, Durbin R. Fast and accurate short read alignment with Burrows-Wheeler transform. Bioinformatics 2009;25:1754-1760.

16 DePristo MA, Banks E, Poplin R et al. A framework for variation discovery and genotyping using nextgeneration DNA sequencing data. Nat Genet 2011;43: 491-498.

17 Cibulskis K, Lawrence MS, Carter SL et al. Sensitive detection of somatic point mutations in impure and heterogeneous cancer samples. Nat Biotechnol 2013;31:213-219.

18 Ramos AH, Lichtenstein L, Gupta M et al. Oncotator: cancer variant annotation tool. Hum Mutat 2015;36: E2423-E2429.

19 Ojesina AI, Lichtenstein L, Freeman SS et al. Landscape of genomic alterations in cervical carcinomas. Nature 2014;506:371-375.
20 Kandoth C, Schultz N, Cherniack AD et al. Cancer Genome Atlas Research NIntegrated genomic characterization of endometrial carcinoma. Nature 2013;497: 67-73.

21 Devouassoux-Shisheboran M, Silver SA, Tavassoli FA. Wolffian adnexal tumor, so-called female adnexal tumor of probable Wolffian origin (FATWO): immunohistochemical evidence in support of a Wolffian origin. Hum Pathol 1999;30:856-863.

22 McCluggage WG, Oliva E, Herrington CS et al. CD10 and calretinin staining of endocervical glandular lesions, endocervical stroma and endometrioid adenocarcinomas of the uterine corpus: CD10 positivity is characteristic of, but not specific for, mesonephric lesions and is not specific for endometrial stroma. Histopathology 2003;43:144-150.

23 Rabban JT, McAlhany S, Lerwill MF et al. PAX2 distinguishes benign mesonephric and mullerian glandular lesions of the cervix from endocervical adenocarcinoma, including minimal deviation adenocarcinoma. Am J Surg Pathol 2010;34:137-146.

24 Yemelyanova A, Gown AM, Wu LS et al. PAX8 expression in uterine adenocarcinomas and mesonephric proliferations. Int J Gynecol Pathol 2014;33: 492-499.

25 Roma AA, Goyal A, Yang B. Differential expression patterns of GATA3 in uterine mesonephric and nonmesonephric lesions. Int J Gynecol Pathol 2015;34: 480-486.

26 Ordi J, Nogales FF, Palacin A et al. Mesonephric adenocarcinoma of the uterine corpus: CD10 expression as evidence of mesonephric differentiation. Am J Surg Pathol 2001;25:1540-1545.

27 Ordi J, Romagosa C, Tavassoli FA et al. CD10 expression in epithelial tissues and tumors of the gynecologic tract: a useful marker in the diagnosis of mesonephric, trophoblastic, and clear cell tumors. Am J Surg Pathol 2003;27:178-186.

28 McConechy MK, Ding J, Cheang MC et al. Use of mutation profiles to refine the classification of endometrial carcinomas. J Pathol 2012;228:20-30.

29 Wright AA, Howitt BE, Myers AP et al. Oncogenic mutations in cervical cancer: genomic differences between adenocarcinomas and squamous cell carcinomas of the cervix. Cancer 2013;119:3776-3783.

30 CeramiE, Gao J, Dogrusoz U et al. The cBio cancer genomics portal: an open platform for exploring multidimensional cancer genomics data. Cancer Discov 2012;2:401-404.

31 Gao J, Aksoy BA, Dogrusoz U et al. Integrative analysis of complex cancer genomics and clinical profiles using the cBioPortal. Sci Signal 2013;6:pl1.

32 Swisher EM, Peiffer-Schneider S, Mutch DG et al. Differences in patterns of TP53 and KRAS2 mutations in a large series of endometrial carcinomas with or without microsatellite instability. Cancer 1999;85: 119-126.

33 Lagarda H, Catasus L, Arguelles R et al. K-ras mutations in endometrial carcinomas with microsatellite instability. J Pathol 2001;193:193-199.

34 Bosse T, ter Haar NT, Seeber LM et al. Loss of ARID1A expression and its relationship with PI3K-Akt pathway alterations, TP53 and microsatellite instability in endometrial cancer. Mod Pathol 2013;26:1525-1535.

35 Olson JM, Hallahan AR. p38 MAP kinase: a convergence point in cancer therapy. Trends Mol Med 2004;10:125-129. 
36 Peyssonnaux C, Eychene A. The Raf/MEK/ERK pathway: new concepts of activation. Biol Cell 2001;93: 53-62.

37 Corcoran RB, Cheng KA, Hata AN et al. Synthetic lethal interaction of combined BCL-XL and MEK inhibition promotes tumor regressions in KRAS mutant cancer models. Cancer Cell 2013;23:121-128.

38 Janne PA, Shaw AT, Pereira JR et al. Selumetinib plus docetaxel for KRAS-mutant advanced non-smallcell lung cancer: a randomised, multicentre, placebocontrolled, phase 2 study. Lancet Oncol 2013;14: $38-47$.

39 Nakayama N, Nakayama K, Yeasmin S et al. KRAS or BRAF mutation status is a useful predictor of sensitivity to MEK inhibition in ovarian cancer. Br J Cancer 2008;99:2020-2028.
40 Engelman JA, Chen L, Tan X et al. Effective use of PI3K and MEK inhibitors to treat mutant Kras G12D and PIK3CA H1047R murine lung cancers. Nat Med 2008;14:1351-1356.

41 Ramalingam SS, Janne PA, Mok T et al. Dacomitinib versus erlotinib in patients with advanced-stage, previously treated non-small-cell lung cancer (ARCHER 1009): a randomised, double-blind, phase 3 trial. Lancet Oncol 2014;15:1369-1378.

42 Chen Z, Cheng K, Walton Z et al. A murine lung cancer co-clinical trial identifies genetic modifiers of therapeutic response. Nature 2012;483:613-617.

43 Hamidi $\mathrm{H}$, Lu M, Chau $\mathrm{K}$ et al. KRAS mutational subtype and copy number predict in vitro response of human pancreatic cancer cell lines to MEK inhibition. Br J Cancer 2014;111:1788-1801.

Supplementary Information accompanies the paper on Modern Pathology website (http://www.nature.com/ modpathol) 